\title{
Línea de investigación: currículo y evaluación educativa*
}

\section{Line of Research: Curriculum and Educational Evaluation}

\author{
José Duván Marín Gallego**
}

Fecha de recepción: 13 de marzo de 2012

Fecha de revisión: 26 de marzo de 2012

Fecha de aprobación: 8 de mayo de 2012

\section{Resumen}

Este artículo desarrolla el concepto de línea de investigación en currículo y evaluación, considerando que la línea se convierte en el eje dinamizador y articulador de los procesos investigativos del Doctorado en Educación de la Universidad Santo Tomás, especialmente para el trabajo de tesis doctoral. Por tanto, la línea así entendida es un sistema estratégico y organizativo que sirve de guía para la acción y facilita el diagnóstico de una problemática en el campo del conocimiento y de la práctica investigativa del currículo y de la evaluación en educación.

* $\quad$ Artículo de reflexión que presenta los resultados de la creación de la línea investigativa de Currículo y Evaluación, perteneciente al Doctorado en Educación de la Universidad Santo Tomás de Bogotá.

** Doctor en Educación, magíster en Evaluación de la Educación, especialista en Docencia Universitaria, abogado, licenciado en Filosofía, docente investigador y coordinador de la Línea de Currículo y Evaluación de la Educación en el Doctorado en Educación de la Universidad Santo Tomás. Correo electrónico: joseduvanmarin@ustadistancia.edu.co 
Palabras clave: Línea, currículo, evaluación, investigación, epistemología, metodología.

\begin{abstract}
This paper develops the concept of a line of research in curriculum and evaluation, considering that the line becomes the main driving force and coordinator of the investigative processes of the Doctorate in Education at the Santo Tomás University, especially for doctoral thesis work. Therefore, the line, so understood, is a strategic and organizational system which can be used as a guide for action and facilitates the diagnosis of a problem in the field of knowledge and practice of curriculum research and evaluation in education.
\end{abstract}

Keywords: Line, curriculum, evaluation, research, epistemology, methodology.

\section{Introducción}

La educación como producto de la sociedad cambiante e histórica, culturalmente situada y dirigida a hombres y mujeres como seres sociales, asume ante sí retos y desafíos de cómo prepararlos para la vida y cómo permitirles enfrentar las situaciones posmodernas en su integralidad. La educación, aún desde las primigenias civilizaciones, ha sido la vía para la formación paulatina de las nuevas generaciones, para su autotransformación y la transformación de la sociedad.

Los procesos educativos giran, sin duda, en torno a los procesos de enseñanza - aprendizaje, especialmente, las formas de cómo las instituciones educativas enseñan, qué enseñan, cómo enseñan, para qué enseñan y a quién enseñan; pero también importan las formas de aprender y lo que las personas necesitan aprender para vivir en este mundo y vivir en sociedad. Todo esto se encierra en el concepto de "currículo"; y el currículo, a la vez, se configura como una selección particular de la cultura. Por tanto, cultura y currículo guardan entre sí una estrecha relación en cuanto a que los contenidos de todo currículo educativo equivalen a una porción selecta de los conocimientos, las creencias y los valores que la comunidad desea conservar y trasmitir a las nuevas generaciones.

Aunque la escuela tradicionalmente no ha sido siempre la institución educadora, sin embargo, en la modernidad y la posmodernidad ha desempeñado el papel más importante en la enseñanza y el aprendizaje de los niños y los adolescentes. Pero también la universidad, desde la Edad Media, ha desempeñado el 
mismo papel importante en la educación superior. De esta manera, aprovechando la trayectoria y experiencia de la Universidad Santo Tomás de casi cuarenta años en la formación de docentes, a lo largo y ancho del territorio nacional, tanto en pregrado como en posgrados (especializaciones y maestrías), esta Línea de Currículo y Evaluación se propone desarrollar procesos de investigación en el Doctorado en Educación, especialmente en las tesis doctorales, si se tiene en cuenta que el currículo y la evaluación de la educación son, quizás, las dos actividades que mayor problemática y debate generan en los procesos pedagógicos, tanto en los aspectos del conocimiento como en la práctica.

De esta problemática, los aspectos que merecen una especial atención para ser investigados son los que se relacionan con la planeación, la construcción y la práctica del currículo, por un lado, y por el otro, las concepciones teóricoepistemológicas, la pertinencia social y la flexibilidad de los currículos, consecuencia de los procesos de globalización y del desarrollo de la ciencia y la tecnología, así como de las nuevas concepciones de política educativa e intereses económicos e industriales que exigen, al mismo tiempo, la necesidad de articular la educación y la pedagogía con los requerimientos de la sociedad. Junto a todos estos problemas, también es necesario investigar acerca de los procesos evaluativos.

En este artículo se desarrollarán los siguientes puntos:
1. El concepto de línea de investigación que se asume para la investigación en currículo y evaluación.

2. Los objetos de investigación de la línea de currículo y evaluación.

3. Los referentes conceptuales y epistemológicos de la línea.

4. Los objetivos y propósitos de la línea.

5. La metodología de investigación en currículo y evaluación educativa.

\section{El concepto de línea en el currículo y la evaluación de la educación}

Antes que cualquier cosa es necesario dilucidar el concepto y la importancia epistemológica y metodológica que la línea reviste para el desarrollo del currículo y la evaluación en el Doctorado en Educación. Para comenzar se plantean dos preguntas fundamentales que permitirán guiar el desarrollo del tema: ¿qué se entiende por línea de investigación? y ¿cómo abordar la construcción de una línea de investigación en currículo y evaluación educativa?

En primer lugar, ya el mismo concepto de línea es tan amplio que se puede aplicar a infinidad de objetos. Se habla, por ejemplo, de línea recta, línea curva, línea telefónica, línea de crédito, línea de parentesco, aprendizaje en línea, entre 
otros. Siguiendo la definición más general de la geometría, una línea es una sucesión continua e indefinida de puntos en una sola dimensión o también suele decirse que la línea recta es la distancia más corta entre dos puntos. Pero, para no caer en ambigüedades y paradojas, es necesario buscar un primer acercamiento a un concepto que coincida con mayor exactitud con el de "línea de investigación"; en este caso y por analogía, se diría que cada punto de una línea de investigación está constituido por una sucesión de estudios, reflexiones sistemáticas, procesos de búsqueda, discusiones y proposiciones que plantean las personas, los grupos y las instituciones que investigan acerca de un tema o de una problemática relacionada con el currículo y la evaluación en educación.

En segundo lugar y de acuerdo con Barrera Morales (2006), una línea de investigación se concreta en una propuesta metodológica y organizacional que orienta el trabajo investigativo y la condición metodológica de personas e instituciones o, también, como la iniciativa que permite hacer visible la actividad científica con criterios de pertinencia, continuidad y coherencia. El mismo autor considera que la línea de investigación se constituye en la expresión continua, multidimensional y compleja, configurada mediante procesos metodológicos orientados hacia la generación de conocimiento.

En tercer lugar, partiendo de las políticas investigativas de la Universidad Santo Tomás, en el Proyecto Institucional Investigativo, PROIN, (2005), se establece que:
Las líneas de investigación constituyen una conceptualización especial y un punto de referencia obligado y básico del modelo investigativo. Dichas líneas se construyen y reconstruyen permanentemente a partir del planteamiento fundamental que se enriquece con los procesos investigativos, con las demandas y necesidades del contexto y con el desarrollo e implementación de los proyectos que dan respuesta a problemas y originan nuevos problemas y nuevos retos dentro de los diversos campos, ante los objetos susceptibles de investigación.

Las líneas de investigación, de acuerdo con el PEI, adquieren un carácter interdisciplinario y transdisciplinario en cuanto se constituyen en el motor que mueve la investigación y en el punto de referencia y de respuesta a las necesidades, programas y propuestas que plantea la investigación en general (p. 82).

La línea se convierte, de esta manera, en el eje dinamizador y articulador de toda la práctica investigativa del Doctorado en Educación, tanto de los proyectos y tesis de los doctorandos como de los grupos de investigación, al mismo tiempo que proporciona una mayor flexibilidad al proceso investigativo en cuanto que, alrededor de una línea, pueden girar varios proyectos y varios grupos y, a la vez, un grupo puede albergar en su interior varias líneas de investigación. La línea así entendida es un sistema estratégico y organizativo que sirve de guía para la acción y facilita el diagnóstico de una problemática en el campo del conocimiento y de la práctica del currículo y de la evaluación.

Por lo general, la línea de investigación nace del interés y de la inquietud académica de indi- 
viduos o grupos de personas que desarrollan un tema o buscan solucionar un problema (Suing, 2008). Al mismo tiempo, se construye como un proceso de trabajo colectivo en un determinado campo del saber, para producir conocimiento en torno a un objeto particular, a partir de la problematización de lo producido hasta el momento y de la elaboración de proyectos específicos de investigación que permitan el avance teórico de ese objeto, el desarrollo de otras formas metodológicas, la formación y consolidación de comunidades académicas y la divulgación de los resultados entre comunidades del saber más amplias (Agudelo, 2004). Además, la línea debe estar apoyada en elementos lógicos, epistemológicos y metodológicos que implican el reconocimiento de aspectos fundamentales, como la identificación de áreas temáticas, la precisión de aspectos de interés particular, la visualización y el despliegue de las potencialidades investigativas que se presentan, a fin de percibir la realidad desde la perspectiva de la ciencia y del conocimiento.

Es preciso destacar el hecho de que la línea debe corresponder a un área de conocimiento lo suficientemente amplia como para que no se agoten los temas de interés que le son propios. Además, todo tema requiere de un trabajo multi y transdisciplinario, lo cual permite varios enfoques alrededor de un mismo aspecto, guardando, sin embargo, la coherencia en torno a unos fundamentos epistemológicos, a una metodología y a una orientación central. Por tanto, los temas y los objetos de conocimiento que puede abordar una línea no se agotan con un problema o con una sola investigación. Pero sí es importante que la línea guarde, en lo posible, una unidad disciplinaria, temática, conceptual, epistemológica y metodológica.

\section{Objetos de investigación y el problema de la demarcación en la línea}

El objeto general de la línea es la investigación en educación y la pedagogía. Sin embargo, es preciso tener en cuenta que la educación tiene discursos de diversa índole no todos científicos, como los de la sociología, la filosofía, la psicología o los políticos, así como las ideologías, solo para nombrar los más comunes. ¿Cuándo se puede, por lo tanto, inferir que el discurso o la teoría en que se ubica un tema o que el autor está construyendo, por ejemplo, en el caso frecuente de las tesis doctorales, es un conocimiento científico? Para Popper, la "demarcación" y el inductivismo fueron los dos problemas fundamentales de la epistemología en el siglo pasado. En efecto, admite Popper (1998) que "casi todas las ciencias empíricas han surgido del seno de la metafísica: su configuración precientífica era especulativo-filosófica. Incluso las ciencias más desarrolladas, como la física, no se han liberado todavía de los restos de su pasado metafísico" (p. 45). Admite también que las ciencias menos desarrolladas como la biología, la psicología o la sociología, han estado y siguen estando dominadas por elementos metafísicos en mayor medida que la física. Se pregunta luego el autor: “¿Se puede justificar este rechazo de la metafísica?, ¿qué se quiere decir exactamente con las expresiones "metafísica" y "ciencia empírica"?, ¿pueden encontrarse diferencias estrictas?, ¿pueden trazarse fronteras perfectamente determinadas?" (p. 46). Aunque el problema 
epistemológico de la demarcación pretende establecer criterios para distinguir un discurso científico de otro no científico, en educación y pedagogía estos límites entre lo propiamente científico y lo filosófico resultan, a veces, casi imposibles de discernir, por la complejidad que caracteriza a estos campos del saber.

No obstante, el problema que plantea la demarcación desde la ciencia clásica en las ciencias sociales y humanas y, concretamente en educación y pedagogía, sí es posible, por lo menos, determinar algunos criterios que nos permitan dar cuenta de un conocimiento con un cierto carácter de rigurosidad científica. Por tanto, para construir un conocimiento válido en estas ciencias es necesario considerar seis elementos básicos, comunes a todas las ciencias sociales y humanas que ayudan, en parte, a identificarlo como científico y a diferenciarlo de otro que no lo sea: el objeto de investigación o de estudio, la epistemología en que se fundamenta ese objeto, la metodología que facilita la reconstrucción y organización del conocimiento, el resultado final de todo el proceso anterior que generalmente se plasma en una teoría, el fin que se propone el conocimiento, el cual debe ser un fin útil y práctico en cuanto busca resolver problemas y satisfacer necesidades de la vida humana y, por último, obtener el aval de una comunidad científica, o como diría Kuhn (1992), de los que practican el mismo paradigma.

Bajo la concepción de la educación y la pedagogía como objetos generales de investigación, el currículo y la evaluación educativa son también objetos específicos y complejos que no pueden ser comprendidos por fuera de los contextos de las transformaciones sociales, de los escenarios nacionales e internacionales, de las atmósferas culturales de cada sociedad, situando en ellas los movimientos, conflictos y contradicciones del conocimiento en evolución y en permanente cambio y, considerando, además, que el objeto fundamental del currículo y la evaluación consiste en integrar diferentes formas de la práctica educativa y pedagógica, diferentes lenguajes y diferentes maneras de construir el conocimiento en estas áreas del saber (Agudelo, 2004).

La línea así planteada centra su atención en el reconocimiento y la reflexión de las teorías y las prácticas del currículo y los procesos de evaluación educativa. Por tanto, a partir del concepto de currículo como todo el aprendizaje que planifica y guía una organización de enseñanza o formación, ya sea que se lleve a cabo individualmente o en grupos, dentro o fuera de un aula de clase, tal como lo señala Taylor (en GUNI, 2008), y que Gimeno Sacristán (2010) considera, por otra parte, como todo lo que sabemos y es potencialmente enseñable y posible de aprender, o como una selección regulada de los contenidos por enseñar y aprender que, a su vez, regula toda la práctica pedagógica (didáctica, evaluación, gestión) que se desarrolla durante la escolaridad; la línea Currículo y Evaluación podrá desarrollar todos aquellos tópicos convergentes y complementarios al eje investigativo de la educación y la pedagogía, como estados del arte, aspectos filosóficos, teóricos y metodológicos; aspectos económicos, políticos y sociales. El concepto de evaluación queda incluido en este mismo contexto, como 
un proceso que debe llevarse a cabo de manera continua y permanente, y que ha de tener como finalidad "el enjuiciamiento sistemático de la valía o el mérito de un objeto" (Stufflebeam y Shinkfield, 1995, p. 19).

Complementario con todo lo anterior, la Ley 115 de 1994, en su artículo 76 y en forma mucho más concisa, define el currículo como:

[...] el conjunto de criterios, planes de estudio; programas, metodologías, y procesos que contribuyen a la formación integral y a la construcción de la identidad cultural nacional, regional y local, incluyendo también los recursos humanos, académicos y físicos para poner en práctica las políticas y llevar a cabo el proyecto educativo institucional.

En esta definición, la Ley 115 describe aspectos tan importantes del currículo que son, sin duda, objetos para ser investigados en un programa de Doctorado en Educación, como la formación integral de la persona y la identidad cultural de la nación, la región y la localidad. De ahí la importancia de la interdisciplinariedad y la transdisciplinariedad de esta línea que facilite el trabajo de proyectos curriculares interculturales, tanto en el orden de lo nacional como de Latinoamérica y el Caribe, pero que a la vez permitan la integración entre regiones, países y culturas.

En resumen y sin pretender caer en una exhaustiva enumeración, la investigación en currículo y evaluación se extiende desde el conocimiento mismo hasta los procesos psicológicos y culturales; las relaciones con otros sistemas y propuestas educativas; los aprendizajes y formas de apropiación del conocimiento; los escenarios, actores y formas de interacción; los medios y mediaciones y la normatividad legal, entre otros. Es por esta razón por la que los temas y las posibilidades de investigación pueden ser inagotables.

Junto a los objetos anteriores podrían añadirse los contextos curriculares, los distintos enfoques, la gestión curricular, objetivos, perfiles, diseños, planes de estudio, actividades curriculares, metodologías, recursos, criterios de evaluación curricular, currículos integrados, etc., así como la planificación, el diseño, implementación y financiación de currículos, además de los currículos comparados, la pertinencia del currículo, los currículos por competencias, el diseño y desarrollo de currículos innovadores y transformadores.

\section{Referentes conceptuales $y$ epistemológicos de la línea de Currículo y Evaluación}

\section{El currículo y la evaluación como prácticas pedagógicas a la luz de los actores}

Cuando nos referimos a las prácticas educativas no debemos entenderlas como mera "actividad" o acción técnica, sino como una construcción con sentido y significación en los planos social, histórico, político y científico que solo puede ser comprendida en su forma interpretativa y crítica (Carr, 1996). Desde esta perspectiva, la práctica pedagógica en la línea de Currículo y Evaluación se fundamenta en los siguientes aspectos: 
1. Una visión antropológica que hace referencia a una concepción filosófica en cuanto a la idea de hombre que se educa, los principios, los fines de la educación y sus valores.

2. El contexto social, referido al modelo de sociedad que se pretende fomentar, en armonía con el bien común, los valores sociales, la participación ciudadana y democrática, la regulación legal y las relaciones sociales que se generan en la institución educativa.

3. Los conocimientos científicos, como la fundamentación epistemológica y metodológica de las diversas áreas y materias que integran el currículo y la evaluación de la educación; la lógica interna de los conocimientos y las relaciones interdisciplinares.

4. Conocimiento psicológico: es el conocimiento acerca de la naturaleza psíquica y humana del estudiante y del docente, tales como sus motivaciones, intereses, necesidades y sus procesos de aprendizaje.

5. Fundamentación pedagógica o fundamentación teórico-práctica que sintetiza la experiencia de los procesos de enseñanzaaprendizaje, sus objetivos, los contenidos, las variables espacio-temporales, las didácticas y los procesos de evaluación, entre otros.

De esta manera, se concibe la educación como un hecho que se desarrolla en las prácticas de la enseñanza-aprendizaje y como proceso activo que ocurre en el interior del estudiante, influido por el docente y por su entorno. Pero, por otra parte, la enseñanza-aprendizaje también es una actividad social que implica interacción entre los participantes y los recursos que utilizan, intercambio de información y un uso prioritario del lenguaje como medio y como acción. Esto permite que el aprendizaje dependa, tanto de la información que el docente presenta al estudiante como del proceso seguido por el discente para asimilar y precisar la información. Por ello, educar significa comunicarse, intercambiar y compartir la vida y el conocimiento.

\section{El currículo y la evaluación a la luz de los saberes}

El eje del Currículo y la Evaluación a la luz de los saberes, enmarcado en esta línea de investigación, se propone aportar elementos teóricos, conceptuales, metodológicos y prácticos, a la dinámica de las estructuras curriculares implicadas en el quehacer académico, en la práctica docente, en los perfiles profesionales y en los procesos de evaluación.

Las reformas de América Latina, en la década del noventa, proyectaron como ejes centrales la cualificación, equidad y pertinencia de la educación y enfatizan, como expresa Tünnermann (1998), en el papel conductor que debe asumir la educación superior en la renovación de todo el sistema social. Como puntos de encuentro, en las distintas reformas, se observa que la transformación curricular se propone como uno de los principales intereses de la política desarrollada por todos los países, con miras a mejorar la calidad de la educación, su pertinencia y proyección en el contexto. 
La política de "Aseguramiento de Calidad" en nuestro país plantea cambios que buscan, desde la transformación curricular y de la evaluación, renovar los programas; diseñar procesos educativos y curriculares interactivos; imponer nuevos modelos de gestión educativa; construir nuevos paradigmas educativos; generar cambios curriculares y evaluativos institucionales de fondo; hacer la educación más pertinente según las necesidades reales del entorno nacional y disciplinar; contextualizar los contenidos propios de los programas y los temas nucleares en la dinámica de cambio que vive el país y el mundo; comprender las complejas relaciones de la comunidad académica; señalar la necesidad de una mayor integración entre las funciones básicas de la universidad: docencia, investigación y extensión.

Las diversas experiencias de transformación y renovación curricular y evaluativas que se llevan a cabo en las instituciones de educación superior reflejan diferencias y características especiales que muestran, no solo la identidad institucional, sino una dinámica cultural en constante recreación y deliberación, al mismo tiempo que exigen asumir los procesos curriculares y evaluativos como procesos investigativos desde sus distintos componentes.

Desde otra perspectiva, las teorías tradicionales del currículo y la evaluación no muestran una articulación entre los procesos. Por el contrario, muestran conformaciones rígidas y atomizadas de los componentes curriculares y evaluativos. Los procesos se presentan divididos en planeación, diseño, construcción, implementación y evaluación, fragmentando aspectos importantes del currículo y dando lugar a estructuras rígidas, cerradas y muchas veces desfasadas de las necesidades personales y de la comunidad, tanto nacional como internacional. Cada componente se contempla y se trabaja como si fuera ajeno a las prácticas pedagógicas, sin comprender que el desarrollo curricular y evaluativo se produce a partir de una acción que debe verse desde una totalidad, en sentido real y que implica, también, discursos formativos. Los currículos y los procesos evaluativos se diseñan pensando solo en un plan de estudios, sin entender las totalidades y las relaciones sistémicas propias de la complejidad de estas ciencias. La evaluación se asume en forma instrumental y técnica, desconectada del diseño curricular, como si se tratara de una identidad académica que se construye dentro de límites cerrados y rígidos de un área de conocimiento, con un agregado de asignaturas descontextualizadas en las cuales está presente el aislamiento entre teoría y práctica, entre conocimiento y aplicación, entre saber y saber hacer, entre conocimiento y problema (Díaz, 2004).

En esta perspectiva, lo que pretende la línea es lograr que los doctorandos comprendan que la investigación en currículo y evaluación tiene que trascender la racionalidad organizativa y académica, para alcanzar la apropiación de un currículo articulado que desarrolle el conocimiento y permita la posibilidad de diálogo e integración entre los saberes y las prácticas académicas, entre la formación integral del educando y la preparación académica, entre el ser y el saber. Además debe ir integrado a la evaluación, como una evaluación sistémica, 
cualitativa y cuantitativa que permita evaluar procesos y condiciones que garanticen la calidad de la educación.

En esta perspectiva, la regulación de la educación superior colombiana enfatiza fuertemente en la estructura curricular y en la evaluación y su transformación. Las nuevas realidades, actores y protagonistas sociales, y los nuevos acontecimientos educativos han obligado a modificar el papel y la función del currículo. Se plantean cambios que buscan reestructurar los programas curriculares con el propósito de cualificar la educación, de hacerla más pertinente a las necesidades reales del entorno nacional y disciplinar; de contextualizar los contenidos propios de los programas y los temas nucleares en la dinámica de cambio que vive el país y el mundo, haciéndolos más pertinentes y flexibles.

En esta dinámica de reflexión y análisis sobre la estructura y la renovación curricular y de los procesos de evaluación surgen varios interrogantes:

- ¿Se soportan las transformaciones curriculares y las nuevas modalidades de práctica pedagógica en los contextos culturales?

- ¿Se asume la problemática curricular desde una visión holística que permita verla en su totalidad, en un movimiento continuo y desde la interacción de sus distintos componentes?

- ¿Se entiende el currículo desde el compromiso con el conocimiento, visto este compromiso desde comunidades científicas que validan conocimiento en distintas áreas del saber?

- ¿Se asume la evaluación como parte imprescindible del proceso curricular?

- ¿Se valoran las disciplinas a partir de la naturaleza de los saberes?, ¿de su lógica interna, de su forma de construcción y apropiación?

- ¿Se tiene en cuenta la evaluación como un paso eminentemente integral, paralelo a todo el proceso de formación implícito en el currículo?

- ¿Qué implicaciones ejercen los factores externos en la generación de ambientes y culturas de los aprendizajes sobre el currículo y la evaluación?

En ese orden de ideas, la línea de Currículo y Evaluación propende porque en los entornos educativos el currículo se asuma como el conjunto de componentes intencionalmente definidos desde el acto pedagógico: actores, saberes, procesos de enseñanza-aprendizaje, formación y contextos. Simultáneamente se asume la evaluación como un proceso integral que mide, avala, evalúa cualitativa y cuantitativamente la calidad de la educación a nivel macro, y cada componente y cada fase a la luz de los procesos educativos y el proyecto curricular. 


\section{El currículo y la evaluación a la luz del contexto científico y tecnológico}

La investigación y la aplicación de las nuevas tecnologías en el campo de la educación y en los procesos pedagógicos se ha convertido en un hecho imperativo, tanto de la formación como de la misma investigación, para lo cual se han ideado estrategias didáctico-pedagógicas específicas que permiten la aplicación en el campo profesional.

El diseño de nuevas tecnologías de la información se caracteriza por el trabajo entre diferentes ámbitos disciplinares y técnicos, cuyo fin es producir nuevos procedimientos didácticos y metodológicos para dar respuesta a problemas de enseñanza y de aprendizaje que se plantean; por tanto, la tecnología articula la técnica, la ciencia, los aspectos socioeconómicos y culturales en los diferentes contextos.

Sin embargo, el conocimiento tecnológico ha adquirido protagonismo, y gran importancia para la producción de nuevas disciplinas y nuevos saberes. En la educación juega un papel importante, pues ayuda a satisfacer las necesidades individuales, ayuda a la profundización del impacto en la sociedad y la economía y a la adopción de innovaciones que cambian el aparato productivo y la interacción social (Castells, 1999). Las TIC han llevado a una nueva revolución, comparable a la revolución industrial del siglo XIX en Inglaterra.

Por tanto, las nuevas tecnologías de la información y de la comunicación brindan conocimientos importantes para la comprensión de medios artificiales, sus características y tendencias, lo cual ayuda, a la vez, a la aparición de nuevos contenidos científicos y tecnológicos, que forman parte de diferentes espacios curriculares, enriqueciéndolos y brindando nuevos contextos para la enseñanza.

La incorporación de tecnologías en los currículos obedece a propósitos científicos y culturales, en el sentido de que la tecnología se incorpora a la cultura y, por tanto, también, a la educación, en la producción de nuevos saberes. En otros términos, lo técnico instrumental está ligado a las capacidades cognitivas, como la resolución de problemas, el diseño de proyectos, de modelos y el uso de equipos novedosos. Alvin Toffler (1993) plantea que se está ante una revolución que ha llevado al nacimiento de las industrias de alta tecnología y novedosos procesos de producción basados en la micro-electrónica. Las tecnologías de la información y de la comunicación provocan un alto impacto en todos los campos de la actividad humana que repercuten directamente en el campo educativo.

Uno de los principales retos desde la línea de Currículo y Evaluación para mejorar la educación en Colombia y en América Latina será el de transformar la información con la cual se encuentra, frecuentemente, en un conocimiento significativo en función de su contexto social.

La presencia e incidencia de las TIC en la vida de los seres humanos y, en particular, de aquellos que hacen parte del ámbito académico, ha generado una situación clara que permite distinguir las diferencias entre aquellos que tienen acceso a altos niveles del conocimiento 
científico y tecnológico (sociedad del conocimiento) y aquellos que están separados del mismo; es importante destacar algunos aspectos de política de ciencia y tecnología e innovación, de gestión de la tecnología, y el papel de las universidades con relación a una conducta del servicio de las ciencias aplicadas

\section{Propósitos y objetivos de la línea}

La línea de Currículo y Evaluación se propone generar procesos investigativos en el ámbito doctoral que impacten en las transformaciones de la educación en Colombia, América Latina y el Caribe y que contribuyan eficazmente a lograr un espíritu de convivencia democrática, de tolerancia y un espíritu de solidaridad y de cooperación, a fin de crear conocimiento, construir la identidad continental y transformar, en forma sostenible, la sociedad.

En forma particular, la línea busca:

1. Desarrollar procesos de conocimiento y de reflexión sistemática y científica en torno al currículo y a la evaluación en educación, en los niveles regional, nacional e internacional.

2. Fomentar y proponer currículos y procesos de evaluación transformacionales y pertinentes, centrados en la reflexión y la acción colectiva que generen impacto en los cambios y transformaciones de las relaciones de poder y en los sistemas socioeconómicos del país y del continente latinoamericano.
3. Proporcionar un apoyo epistemológico y metodológico a los proyectos de investigación y a las tesis de grado de los estudiantes del Doctorado en Educación.

\section{Metodología de la línea de Investigación Currículo y Evaluación}

Cada vez se abre un nuevo campo de investigación y de conocimiento en el que, como dice ASCOFADE (2006) "tiene que ver fundamentalmente con el reconocimiento de la Educación [y la pedagogía], como un campo científico con identidad propia, que avanza de manera significativa en su consolidación en el país" (p. 7). Desde el siglo pasado se ha asistido al surgimiento y despliegue de una nueva generación de reformas educativas y pedagógicas relacionadas con la calidad, la eficiencia, la eficacia y la equidad del servicio que se presta a la comunidad. La educación y la pedagogía trabajan con una materia prima que es, por un lado, el estudiante y, por el otro, el conocimiento. El estudiante como sujeto de formación y el conocimiento como objeto de enseñanza-aprendizaje. Ambos, dentro de este contexto, adquieren un significado y un valor especial porque poseen dos características sui generis: la "educabilidad" y la "enseñabilidad". El término "educabilidad" fue usado por Herbart para significar la capacidad radical del ser humano de perfeccionarse y autorrealizarse (Fermoso Estébanez, 1991) y la "enseñabilidad" se entiende como ese constitutivo intrínseco de la estructura de la ciencia y de todas las disciplinas en general, de ser comunicadas y de ser enseñadas. En otros términos, el conocimiento 
debe ser reducido a procesos pedagógicos y metodológicos para que sea comprendido y aprendido por el estudiante, tarea que debe realizar el docente en el acto de la enseñanzaaprendizaje (Flórez Ochoa, 1994).

Por otra parte, el conocimiento que se enseña y se aprende en la educación y en los procesos pedagógicos (entendiendo estos últimos como procesos de enseñanza y aprendizaje) adquiere una connotación muy particular frente al conocimiento que se produce y se maneja en otras instancias y en otros círculos, tal como ocurre, por ejemplo, con el conocimiento producido en un laboratorio farmacéutico, en un instituto de investigación o en una empresa industrial, el cual se produce, se utiliza y se divulga con fines comerciales, mientras que el conocimiento que se recoge, se genera o se produce en una institución educativa o en un programa académico, como es el caso del Doctorado en Educación, además de poder ser divulgado, debe producirse, especialmente, para ser enseñado y para transformar una realidad que es cada vez más compleja. En otras palabras, este conocimiento se mediatiza por el contexto, las teorías y las prácticas pedagógicas, y por tanto hay que reducirlo a categorías, metodologías y estrategias que lo hagan comprensible a las personas que están en proceso de formación y aprendizaje.

El aspecto anterior constituye el núcleo esencial de esta línea de investigación porque es en ese contexto de la educabilidad y la enseñabilidad en el que se generan todos los problemas relacionados con la epistemología y la metodología del currículo y la evaluación.
Desde esta perspectiva es importante pensar en el desarrollo de un modelo metodológico de investigación para la línea que nos ocupa. Este modelo está fundamentado en un enfoque complejo de la educación y la pedagogía y, por ello, también, del currículo y la evaluación educativa. Es necesario entender la complejidad en la forma como la describe Edgar Morín (1998, p. 59):

\begin{abstract}
Es con Wiener y Ashby, los fundadores de la Cibernética, [que la complejidad] entra verdaderamente en escena en la ciencia. Es con von Neumann que, por primera vez, el carácter fundamental del concepto de complejidad aparece enlazado con los fenómenos de auto-organización.
\end{abstract}

A renglón seguido se pregunta Morin.

¿Qué es la complejidad? A primera vista,
es un fenómeno cuantitativo, una cantidad
extrema de interacciones e interferencias
entre un número muy grande de unidades.
[...] Pero la complejidad no comprende so-
lamente cantidades de unidades e interac-
ciones que desafían nuestras posibilidades
de cálculo; comprende también incertidum-
bres, indeterminaciones, fenómenos aleato-
rios. En un sentido, la complejidad siempre
está relacionada con el azar.

La incertidumbre que describe Morin no solamente se halla instaurada en los límites de nuestro entendimiento y en la comprensión de la realidad, sino también en los fenómenos mismos, la que, a su vez, "se halla ligada a una cierta mezcla de orden y de desorden" (p. 60). En efecto, la ciencia ya no es ciencia de las certezas, sino de lo complejo y, por tanto, de lo azaroso, de las incertidumbres, de un mundo de inestabilidades acompañadas de orden y 
desorden, de las relaciones dialécticas y de lo meramente probable. Bajo estos presupuestos de la complejidad, el modelo metodológico de investigación en la línea de currículo y evaluación ya no puede reducirse a un solo enfoque, a un único método de investigación o a un modelo reduccionista.

El modelo metodológico de investigación en la línea de currículo y evaluación recoge, en consecuencia, las siguientes características:

1. Implica un diseño metodológico que trabaje a partir de cuestionamientos, problemas y proyectos y que acepte la multicomplejidad y la pluralidad epistemológica y metodológica como referentes inmediatos.

2. Pretende, como marco de referencia general, la elaboración y discusión de hechos, documentos, relaciones, interacciones y retrodicciones, tanto en las etapas de configuración y consolidación como en las de desarrollo de los problemas inherentes a la investigación del currículo y la evaluación en educación.

3. Considera la información como el proceso de diferenciación significativa y fundamental en la investigación, por lo cual reconoce y acepta la diversidad de fuentes y métodos de recolección, estrategias y análisis, a la vez que exige la permanente producción y difusión de material escrito, a partir de la base de la investigación.

4. Se apoya en el carácter inter, multi y transdisciplinario de la investigación en educación y pedagogía, acudiendo a las especificidades de los proyectos de las tesis de cada doctorando.

5. Reconoce las hipótesis, los cuestionamientos, las preguntas y los problemas como puntos de partida y partes nucleares, iniciales del proceso investigativo, los cuales dan cabida a los proyectos.

6. El modelo contempla lo global ${ }^{1}$ como uno de los ejes que garantiza un sistema de referencia, junto con lo multidimensional y lo complejo. En consecuencia, la investigación debe promover una búsqueda del conocimiento y una producción del mismo, referido al contexto multidimensional, dentro de un eje que considera el conocimiento universal y una diversidad del saber.

7. El modelo considera los alcances de las políticas educativas, los valores humanos, sociales y éticos de la educación y la pedagogía, así como la estructura orgánica investigativa, los niveles de acción, sus

1 El término "global" corresponde a un término de moda, pero también a una problemática que surge en el siglo $\mathrm{XV}$ cuando se empieza a concebir la tierra como un todo, hasta los modernos avances telecomunicativos. Ander-Egg sintetiza en su obra Reflexiones en torno al proceso de mundialización/globalización como algo que acontece en todo el mundo y que afecta, de una u otra manera, a cada ser y a toda la población mundial. Se habla de Globalización económica, política, cultural. Siendo optimistas, añade Ander-Egg: "no debemos vivir esta situación como un problema (o varios y complejos problemas como en verdad lo es), sino también como una posibilidad; en sentido estricto, como posibilidades múltiples $\mathrm{y}$, a su vez, ambivalentes en cuanto a sus oportunidades para un mayor desarrollo humano y mejor calidad de vida" (p. 9). 
componentes, los sistemas, las redes y los grupos de investigación.

La metodología compleja admite como válidos metodológica y epistemológicamente los diversos paradigmas de orden cualitativo y cuantitativo; los métodos que se adscriben a cada uno de estos paradigmas; así como las técnicas y estrategias de recogida y análisis de la información. Por tanto, serán válidos los métodos cuantitativos de observación, experimentación, cuasi experimentación; los métodos correlacionales, el estudio de caso, etc. En relación con los métodos cualitativos: la etnografía, la etnometodología, la investigación acción, la teoría fundamentada, el estudio de caso cualitativo, la historia de vida y otros métodos alternativos pertinentes.

Sin embargo, ante la variedad de métodos y ante la complejidad del conocimiento, el proceso de investigación deberá contemplar tres momentos esenciales: un momento de búsqueda (heurística), un momento de organización y sistematización de la información (metodología) y un momento de construcción de conocimiento (teoría).

Tampoco la complejidad excluye los siguientes pasos metodológicos en la construcción del conocimiento y en la elaboración de la tesis doctoral acerca del currículo y de la evaluación:

1. El planteamiento del problema.

2. La fundamentación teórica o marcos de referencia.
3. El proceso de recogida y análisis de la información.

4. La elaboración y construcción de la tesis.

\section{Conclusiones}

De acuerdo con el Documento Maestro del Programa de Doctorado en Educación de la Universidad Santo Tomás (2009), para este Doctorado:

[...] todo currículo acontece en una cultura, lugar y tiempo concretos; por lo tanto, el currículo es el instrumento, quizás el más valioso, del que disponen las instituciones de educación para contribuir al cambio y al desarrollo social, pues, la educación tiene un papel cada vez más importante, en todos los niveles, como transmisora y reproductora de un complejo tejido de conocimientos, valores, relaciones sociales $y$ de poder (p. 74)

De esta manera, la investigación en currículo y evaluación, tal como se propone en la línea, deberá tener un enorme impacto, no solamente en el desarrollo de los individuos, sino en las transformaciones de la educación en Colombia y América Latina, hacia donde está proyectado el Doctorado en Educación y, por ende, también en toda la sociedad.

La señora Hebe Vessuri (en GUNI, 2008) sostiene que "en los países más débiles, los esfuerzos por poner la ciencia y la tecnología al servicio del desarrollo humano han arrojado resultados terribles. La globalización tal como la conocemos hoy en día es fundamentalmente 
asimétrica" (pp. 121-122). Nos preguntaríamos entonces, ¿cuál será la causa de esta asimetría? Sin duda la primera y principal es la poca inversión de los países pobres y de sus instituciones en investigación científica y tecnológica; pero, por otra parte, también la falta de investigadores que realicen investigaciones de punta y de impacto en los distintos campos requeridos por la comunidad. Una sociedad como la nuestra, con necesidades de distinto orden, deberá hacer un esfuerzo, en primer lugar, por formar buenos investigadores en los niveles de maestría y doctorado y, en segundo lugar, brindarles el apoyo necesario, económico, logístico y de tiempo, para realizar las investigaciones acerca de los problemas prioritarios del país. Sin duda, uno de los problemas más urgentes es el de la educación, como el eje principal del desarrollo humano sostenible.

La línea de Investigación de Currículo y Evaluación se propone formar investigadores en este campo de la educación, proporcionándoles, por lo menos, a los estudiantes del Doctorado en Educación de la Universidad Santo Tomás, algunas herramientas epistemológicas y metodológicas para hacer investigación en educación y pedagogía que contribuyan con la innovación y la transformación que requieren los niños (as) y jóvenes de Colombia, teniendo en cuenta que, de acuerdo con el artículo 13 de la Ley 30 de 1992: "Los programas de doctorado se concentran en la formación de investigadores a nivel avanzado tomando como base la disposición, capacidad y conocimientos adquiridos por la persona en los niveles anteriores de formación. El doctorado debe culminar con una tesis".

\section{Referencias}

Agudelo, N. (2004, julio-diciembre). Las líneas de investigación y la formación de investigadores: una mirada desde la administración y sus procesos formativos. Revista Electrónica de la Red de Investigación, 1 (1). Consultada el 6 de julio de 2010 desde: http://revista.iered.org

ASCOFADE (2006). Reflexiones en torno a la reforma de Colciencias: documento "Área de la educación, la cultura y las instituciones". Bogotá: ASCOFADE.

Carr, W. (1996). Una teoría para la educación. Hacia una investigación educativa crítica. Madrid: Morata.

Castells, M. (1999). La era de la información: economía, sociedad y cultura (3 vols.). Madrid: Alianza Editorial.

Gimeno Sacristán, J. (Comp.). (2010). Saberes e incertidumbres sobre el currículum. Madrid: Morata.

Kuhn, T. (1992). La estructura de las revoluciones científicas (1 ${ }^{\text {a }}$. reimp.). Bogotá: Fondo de Cultura Económica.

Morin, E. (1998). Introducción al pensamiento complejo (2a . ed.). Barcelona: Gedisa. 
Popper, K.R. (1998). Los dos problemas fundamentales de la epistemología. Basado en manuscritos de los años 1930-1933. Madrid: Tecnos.

Suing, A. (2008). Definición de la línea de investigación. Compilación para insumo del CITTES de Comunicación de la UTPL. Establecimiento de líneas y temas de investigación. Universidad Técnica Particular de Loja. Recuperado de internet, 8 de julio de 2010, desde: http://www.abelsuing.files. wordpress.com/2008/07/que-es-una-lineade-investigacion.doc

Stufflebeam, D.L. y Shinkfield, A.J. (1995). EvaIuación sistemática. Guía teórica y práctica (3a. reimp.). Barcelona: Paidós.

Taylor, P. (2008). El currículo de la educación superior para el desarrollo humano y social. En: Educación Superior en el mundo 3. Educación Superior: nuevos retos y roles emergentes para el desarrollo humano y social. (pp. 89-100). Barcelona: Global University Network For Innovation. Mundiprensa.
Toffler, A. (1993). La Tercera Ola. Barcelona: Plaza \& Janés.

Tünnermann, C. (1998). Tendencias innovativas en la educación superior. En Políticas y estrategias para la transformación de la educación superior en América Latina y el Caribe. Bogotá: ASCUN, UNESCO, CRESAL.

Universidad Santo Tomás. (2005). Proyecto Investigativo Institucional, PROIN. Bogotá: Publicaciones USTA.

Universidad Santo Tomás (2009). Doctorado en Educación. Condiciones del Programa. Condiciones Institucionales. Bogotá.

Vessuri, H. (2008). El rol de la investigación en educación superior: implicaciones y desafíos para contribuir activamente al desarrollo humano y social. En Educación superior en el mundo 3. Educación superior: nuevos retos y roles emergentes para el desarrollo humano y social. (119-129). Barcelona: Global University Network For Innovation. Mundiprensa. 